\title{
Eventos cardiovasculares relacionados con el uso de macrólidos en una unidad de cuidado intensivo
}

\section{Cardiovascular events related to the use of macrolides in an intensive care unit}

\author{
Abraham Alí-Munive, Leidy Paola Prada-Romero, Emily Rincón-Álvarez, \\ Ángela Hernández-Parra • Bogotá, D.C. (Colombia)
}

DOI: https://doi.org/10.36104/amc.2020.1336

\section{Resumen}

Introducción y objetivos: los macrólidos son antibióticos ampliamente utilizados con los cuales se ha reportado una mayor frecuencia de eventos cardiovasculares relacionados con aumento de arritmias. Mediante este estudio, se busca describir algunas complicaciones cardiovasculares con el uso de macrólidos en pacientes en UCI.

Materiales y métodos: se realizó un estudio descriptivo de corte transversal en el que se incluyeron pacientes adultos que ingresaron a la unidad de cuidado intensivo médico de la Fundación Cardioinfantil y que recibieron tratamiento antibiótico con claritromicina durante los años 2013 y 2015.

Resultados: la muestra recolectada fue de 38 pacientes, la mediana de edad fue de 64 años y la claritromicina se usó más frecuentemente en el tratamiento de infecciones adquiridas en la comunidad, siendo la neumonía el diagnóstico más común. La frecuencia de arritmias tipo fibrilación o flutter auricular fue de $7.89 \%$ y de taquicardia ventricular $2.63 \%$. El medicamento concomitante más frecuentemente usado fue la quetiapina con $28.95 \%$. La principal causa de muerte fue falla respiratoria.

Conclusiones: la frecuencia de arritmias fue alta en nuestro, aunque la causa de muerte más frecuente fue falla respiratoria. (Acta Med Colomb 2020; 45. DOI: https://doi.org/10.36104/ amc.2020.1336).

Palabras clave: macrólido, claritromicina, neumonía, fibrilación atrial, enfermedades cardiovasculares.

\section{Abstract}

Introduction and objectives: macrolides are widely used antibiotics for which a greater frequency of cardiovascular events related to increased arrhythmias has been reported. This study seeks to describe some cardiovascular complications of the use of macrolides in ICU patients.

Materials and methods: this was a descriptive cross-sectional study which included adult patients admitted to the Medical Intensive Care Unit at the Fundación Cardioinfantil who received antibiotic treatment with clarithromycin in 2013 and 2015.

Results: the collected sample was 38 patients. The median age was 64 years, and clarithromycin was most frequently used for treating community-acquired infections, with pneumonia being the most common diagnosis. The frequency of atrial fibrillation or flutter was $7.89 \%$, and ventricular tachycardia $2.63 \%$. The most frequently used concomitant medication was quetiapine at $28.95 \%$. The main cause of death was respiratory failure.

Conclusions: the frequency of arrhythmias was high in our study, although the most frequent cause of death was respiratory failure. (Acta Med Colomb 2020; 45. DOI: https://doi.org/10.36104/ amc.2020.1336).

Key words: macrolide, clarithromycin, pneumonia, atrial fibrillation, cardiovascular diseases.
Dr. Abraham Alí-Munive: Neumólogo - Intensivista Fundación Neumológica Colombiana, Fundación Cardioinfantil. Profesor Titular Universidad del Rosario, Profesor Clínico Principal Universidad de la Sabana; Dra. Leidy Paola Prada-Romero: Especialista en Medicina Interna y Epidemiología Clínica. Médico Internista, Fundación Neumológica Colombiana; Dra. Emily Rincón-Álvarez: InternistaNeumóloga Fundación Neumológica Colombiana, Fundación Cardioinfantil; Dra. Angela Hernández-Parra: Especialista en Medicina Crítica y Cuidado Intensivo, Epidemióloga Clínica Palermo y Hospital Universitario Mayor MEDERI. Bogotá, D.C. (Colombia).

Correspondencia: Dr. Abraham Alí-Munive. Bogota, D.C. (Colombia).

E-mail: aali@neumologica.org

Recibido: 23/IV/2019 Aceptado: 17/III/2020 


\section{Introducción y objetivos}

Los macrólidos son antibióticos ampliamente utilizados en el manejo de varias enfermedades entre ellas las infecciones respiratorias. El prototipo de este grupo es la eritromicina cuyo primer uso clínico en infecciones del tracto respiratorio superior ocurrió en los años 50 (1).

Además de los efectos antimicrobianos de los macrólidos, se ha documentado que tienen propiedades antiinflamatorias e inmunomoduladoras $(1,2)$. Dado la amplia utilización de este grupo de antibióticos en los últimos 15 años, se han reportado eventos cardiovasculares relacionados con su uso. Dentro de éstos se ha descrito la prolongación del intervalo QT, arritmias como torsades de pointes y taquicardia ventricular, y muerte súbita de origen cardiaco (3-5).

En la unidad de cuidado intensivo médico de la Fundación Cardioinfantil - Fundación Neumológica Colombiana la sepsis pulmonar fue la primera causa de ingreso para el año 2012 y la neumonía adquirida en comunidad representó el 33\% de los casos. Para el manejo de esta patología está indicado el uso de claritromicina según las guías de manejo nacionales. No se conocen los posibles efectos cardiovasculares para esta población, razón por la cual se hace necesario determinar las complicaciones asociadas a los eventos cardiovasculares relacionados con el uso de macrólidos en ese grupo de pacientes.

\section{Metodología}

Se realizó un estudio descriptivo de corte transversal en el que se incluyeron pacientes mayores de 18 años que ingresaron a la unidad de cuidado intensivo médico (UCIM) de la Fundación Cardioinfantil y que recibieron tratamiento antibiótico con claritromicina durante los años 2013 y 2015.

\section{Criterios de selección}

- Criterios de inclusión: pacientes mayores de 18 años, ingreso a UCIM, tratamiento con claritromicina.

- Criterios de exclusión: evento cardiovascular agudo al ingreso a UCIM, criterios de no reanimación, hepatopatía severa, posoperatorios de trasplante de órgano sólido, requerimiento de dos o más vasopresores o inotrópicos.

A todos los pacientes que cumplieron los criterios de selección se les realizó seguimiento electrocardiográfico diario desde el ingreso a la UCIM hasta el día quinto, y si ocurría un episodio de taquiarritmia aguda.

\section{Plan de análisis de los datos}

Posterior a la recolección de los datos, se verificó que los datos registrados correspondieran con el tipo de variable, su unidad correspondiente y la codificación en caso de aplicarse. Se realizó una descripción de la muestra utilizando medidas de tendencia central de las variables de naturaleza cuantitativa, así mismo se calcularon las proporciones de las variables de naturaleza cualitativa. Para el análisis de los datos se utilizó el software estadístico STATA 11.

\section{Consideraciones éticas}

El protocolo fue aprobado por la Comité de Ética en Investigación de la Fundación Cardioinfantil.

\section{Resultados}

Durante el periodo del estudio se recolectó una muestra de 38 pacientes, de los cuales $50 \%$ eran mujeres y la mediana de edad fue de 64 años. Con respecto a la distribución de las comorbilidades, la enfermedad respiratoria fue la más frecuente con $63.16 \%$ de los pacientes, seguida por hipertensión arterial con $47.37 \%$ y enfermedad cardiaca con $31.58 \%$. Las comorbilidad menos frecuente fue cáncer el cual fue reportado en sólo uno de los pacientes (Tabla 1).

El diagnóstico más frecuente fue neumonía (97.37\%) y en 37 casos las infecciones fueron adquiridas en la comunidad. La mediana del puntaje de APACHE II al ingreso de los pacientes fue 10.5 (rango intercuartílico 5-17) y la mediana de puntaje de SOFA al inicio fue de 5 (rango intercuartílico 3-9). La mortalidad intrahospitalaria en nuestra muestra de pacientes fue $21.05 \%$ que corresponde a ocho pacientes.

Tabla 1. Características iniciales de los pacientes.

\begin{tabular}{|c|c|}
\hline Característica & $\mathbf{n}=\mathbf{3 8}$ \\
\hline Edad en años, mediana (Q1 , Q3) & $64(52,77)$ \\
\hline Mujeres, no (\%) & $19(50)$ \\
\hline \multicolumn{2}{|l|}{ Comorbilidades } \\
\hline Enfermedad respiratoria, no (\%) & $24(63.16)$ \\
\hline Hipertensión arterial, no (\%) & $18(47.37)$ \\
\hline Enfermedad cardiaca, no (\%) & $12(31.58)$ \\
\hline Enfermedad renal, no (\%) & $7(18.42)$ \\
\hline Diabetes mellitus, no (\%) & $7(18.42)$ \\
\hline Enfermedad neurológica, no (\%) & $4(10.53)$ \\
\hline Enfermedad autoinmune, no (\%) & $2(5.26)$ \\
\hline Cáncer, no (\%) & $1(2.63)$ \\
\hline \multicolumn{2}{|l|}{ Diagnóstico } \\
\hline Neumonía, no (\%) & $37(97.37)$ \\
\hline Traqueobronquitis, no (\%) & $1(2.63)$ \\
\hline \multicolumn{2}{|l|}{ Origen de la infección } \\
\hline Adquirida en la comunidad, no (\%) & $37(97.37)$ \\
\hline Nosocomial, no (\%) & $1(2.63)$ \\
\hline \multicolumn{2}{|l|}{ Estado del paciente al ingreso } \\
\hline Puntaje APACHE II, mediana (Q1, Q3) & $10.5(5,17)$ \\
\hline Puntaje SOFA inicio, mediana (Q1, Q3) & $5(3,9)$ \\
\hline \multicolumn{2}{|l|}{ Desenlaces } \\
\hline Mortalidad, no (\%) & $8(21.05)$ \\
\hline
\end{tabular}


Con respecto al manejo durante la estancia en la unidad de cuidado intensivo, la mediana de estancia en UCI fue de 4.5 días, el $57.89 \%$ de los pacientes requirieron soporte vasopresor y $65.79 \%$ requirieron ventilación mecánica. Al analizar la presencia de eventos cardiovasculares, se documentó que la mediana del intervalo QT corregido inicial fue de 447 milisegundos, siendo un valor similar a la mediana del intervalo QT corregido final de 452 milisegundos. En 7.89\% de los pacientes se presentó fibrilación o flutter auricular y en 1 caso se presentó taquicardia ventricular sostenida (Tabla 2).

La terapia antibiótica combinada usada como primera línea con claritromicina fue con ampicilina/sulbactam $55.26 \%$, piperacilina/tazobactam $21.05 \%$ y cefepime $15.79 \%$. Los menos utilizados fueron meropenem, vancomicina y moxifloxacino (Tabla 3).

Contamos con el dato de los valores de los electrolitos séricos de 36 pacientes durante su estancia en UCI. El $30.56 \%$ presentaban hiperkalemia, $58.33 \%$ hiponatremia, $66.67 \%$ hipomagnesemia y $19.44 \%$ hipocalcemia. También se analizaron los medicamentos de uso concomitante, la quetiapina se usó en $28.95 \%$ de los pacientes, la fluoxetina en $21.05 \%$, la amiodarona en $15.79 \%$ y las quinolonas sólo se usaron en dos casos (Tabla 4).

Al realizar un análisis detallado de las características de los 11 pacientes que recibieron manejo concomitante con quetiapina, se encontró que no hubo diferencias significativas en la mediana del intervalo QT corregido inicial y final, y no se presentaron casos de arritmias auriculares

Tabla 2. Características del manejo en UCI.

\begin{tabular}{|l|c|}
\hline \multicolumn{1}{|c|}{ Variable } & $\mathbf{n = 3 8}$ \\
\hline $\begin{array}{l}\text { Estancia en unidad de cuidado intensivo en días, } \\
\text { mediana (Q1, Q3) }\end{array}$ & $4.5(2,12)$ \\
\hline Requerimiento soporte vasopresor, no (\%) & $22(57.89)$ \\
\hline Requerimiento ventilación mecánica, no (\%) & $25(65.79)$ \\
\hline Lactato elevado, no (\%) & $13(34.21)$ \\
\hline Intervalo QT corregido inicial, mediana (Q1, Q3) & $447 \mathrm{~ms} \mathrm{(438,453)}$ \\
\hline Intervalo QT corregido final, mediana (Q1, Q3) & $452 \mathrm{~ms} \mathrm{(442,481)}$ \\
\hline Fibrilación auricular/flutter auricular, no (\%) & $3(7.89)$ \\
\hline Taquicardia ventricular sostenida, no (\%) & $1(2.63)$ \\
\hline
\end{tabular}

Tabla 3. Antibióticos usados como primera línea.

\begin{tabular}{|l|c|}
\hline \multicolumn{1}{|c|}{ Antibiótico } & $\mathbf{n = 3 8}$ \\
\hline Ampicilina/sulbactam, no. (\%) & $21(55.26)$ \\
\hline Piperacilina/tazobactam, no. (\%) & $8(21.05)$ \\
\hline Cefepime, no. (\%) & $6(15.79)$ \\
\hline Meropenem, no. (\%) & $1(2.63)$ \\
\hline Vancomicina, no. (\%) & $1(2.63)$ \\
\hline Moxifloxacino, no. (\%) & $1(2.63)$ \\
\hline
\end{tabular}

ni ventriculares. $\mathrm{Al}$ analizar los electrolitos séricos de los 10 pacientes de este grupo de quienes teníamos estos datos disponibles, entre 50 y $70 \%$ tenían el potasio, el magnesio y el calcio dentro de rangos normales. El $40 \%$ de estos pa-

Tabla 4. Valores de electrolitos séricos y medicamentos concomitantes.

\begin{tabular}{|l|c|}
\hline \multicolumn{1}{|c|}{ Electrolitos séricos } & $\mathbf{n}=\mathbf{3 8}$ \\
\hline Hiperkalemia, no (\%) & $11(30.56)$ \\
\hline Hipokalemia, no (\%) & $5(13.89)$ \\
\hline Hipernatremia, no (\%) & $8(22.22)$ \\
\hline Hiponatremia, no (\%) & $21(58.33)$ \\
\hline Hipermagnesemia, no (\%) & $8(22.22)$ \\
\hline Hipomagnesemia, no (\%) & $4(11.11)$ \\
\hline Hipercalcemia, no (\%) & $10(27.78)$ \\
\hline Hipocalcemia, no (\%) & $7(19.44)$ \\
\hline Medicamento & $\mathbf{n = 3 8}$ \\
\hline Amiodarona, no (\%) & $6(15.79)$ \\
\hline Fluoxetina, no (\%) & $8(21.05)$ \\
\hline Quetiapina, no (\%) & $11(28.95)$ \\
\hline Quinolonas, no (\%) & $2(5.26)$ \\
\hline
\end{tabular}

Tabla 5. Datos EKG y electrolitos uso concomitante claritromicina y quetiapina.

\begin{tabular}{|c|c|}
\hline Datos electrocardiográficos & $\mathbf{n}=\mathbf{1 1}$ \\
\hline Intervalo QT corregido inicial, mediana (Q1, Q3) & $441.5 \mathrm{~ms}(420,447)$ \\
\hline Intervalo QT corregido final, mediana (Q1, Q3) & $446.5 \mathrm{~ms}(430,481)$ \\
\hline Fibrilación auricular/Flutter auricular, no (\%) & $0(0)$ \\
\hline Taquicardia ventricular sostenida, no (\%) & $0(0)$ \\
\hline Potasio sérico & $\mathbf{n}=\mathbf{1 0}$ \\
\hline Alto, no (\%) & $3(30)$ \\
\hline Bajo, no (\%) & $2(20)$ \\
\hline Normal, no (\%) & $5(50)$ \\
\hline Sodio sérico & $\mathbf{n}=\mathbf{1 0}$ \\
\hline Alto, no $(\%)$ & $3(30)$ \\
\hline Bajo, no (\%) & $4(40)$ \\
\hline Normal, no (\%) & $3(30)$ \\
\hline Magnesio sérico & $\mathbf{n}=\mathbf{1 0}$ \\
\hline Alto, no (\%) & $2(20)$ \\
\hline Bajo, no (\%) & $1(10)$ \\
\hline Normal, no (\%) & $7(70)$ \\
\hline Calcio sérico & $\mathrm{n}=\mathbf{1 0}$ \\
\hline Alto, no (\%) & $2(20)$ \\
\hline Bajo, no (\%) & $2(20)$ \\
\hline Normal, no (\%) & $6(60)$ \\
\hline
\end{tabular}


Tabla 6. Casos que presentaron arritmias durante su estancia en UCI

\begin{tabular}{|c|c|c|c|c|c|c|c|c|c|c|}
\hline & $\begin{array}{l}\text { Edad } \\
\text { (Años) }\end{array}$ & $\begin{array}{c}\text { Arritmia } \\
\text { presentada }\end{array}$ & $\begin{array}{c}\text { Enfermedad } \\
\text { cardiaca de } \\
\text { base }\end{array}$ & $\begin{array}{c}\text { Intervalo } \\
\text { QTc inicial }\end{array}$ & $\begin{array}{l}\text { Intervalo } \\
\text { QTe final }\end{array}$ & Sodio & Potasio & Magnesio & Calcio & $\begin{array}{l}\text { Medicamentos } \\
\text { concomitantes }\end{array}$ \\
\hline Caso 1 & 72 & $\begin{array}{c}\text { Fibrilación } \\
\text { auricular }\end{array}$ & Sí & $520 \mathrm{~ms}$ & $534 \mathrm{~ms}$ & Alto & Normal & Alto & Normal & $\begin{array}{c}\text { Amiodarona, } \\
\text { fluoxetina y } \\
\text { quinolona }\end{array}$ \\
\hline Caso 2 & 78 & $\begin{array}{l}\text { Flutter } \\
\text { auricular }\end{array}$ & Sí & $471 \mathrm{~ms}$ & $471 \mathrm{~ms}$ & Normal & Bajo & Normal & Alto & Ninguno \\
\hline Caso 3 & 74 & $\begin{array}{c}\text { Fibrilación } \\
\text { auricular }\end{array}$ & Sí & $495 \mathrm{~ms}$ & $480 \mathrm{~ms}$ & Normal & Bajo & Normal & Alto & Amiodarona \\
\hline Caso 4 & 81 & $\begin{array}{c}\text { Taquicardia } \\
\text { ventricular no } \\
\text { sostenida }\end{array}$ & Sí & $540 \mathrm{~ms}$ & $480 \mathrm{~ms}$ & Alto & Bajo & Alto & Alto & Ninguno \\
\hline
\end{tabular}

Tabla 7. Causas de muerte.

\begin{tabular}{|l|c|}
\hline \multicolumn{1}{|c|}{ Causas de muerte intrahospitalaria } & $\mathbf{n = 8}$ \\
\hline Falla respiratoria, no (\%) & $4(50)$ \\
\hline Disfunción multiorgánica, no (\%) & $2(25)$ \\
\hline Shock hipovolémico, no (\%) & $1(12.5)$ \\
\hline Arritmia ventricular, no (\%) & $1(12.5)$ \\
\hline
\end{tabular}

cientes tenían hiponatremia y $30 \%$ tenían el sodio en rango normal (Tabla 5).

Se analizaron las variables de mayor interés de los cuatro casos que presentaron algún tipo de arritmia durante la estancia en UCI. Los cuatro pacientes tenían enfermedad cardiaca de base y todos presentaban tanto el intervalo QTc inicial como el final por encima del límite de la normalidad. Sólo en dos casos estaban en tratamiento con medicamentos concomitantes y la mayoría de ellos tenían hipokalemia (Tabla 6).

Finalmente se analizaron las causas de muerte de los ocho pacientes que fallecieron intrahospitalariamente, siendo la causa más frecuente la falla respiratoria (50\%). Sólo un caso falleció por presencia de arritmia ventricular que corresponde al mismo paciente que presentó el evento de taquicardia ventricular sostenida durante su estancia en UCI y de quien se presenta la información relevante en la Tabla 6, caso 4 . También se reportaron fallecimientos por disfunción multiorgánica y shock hipovolémico (Tabla 6).

\section{Discusión}

Los macrólidos corresponden a los antibióticos producidos por hongos del género Streptomyces y algunas bacterias como Arthrobacter y se componen por un anillo macrocíclico de lactona. El prototipo de este grupo es la eritromicina cuyo primer uso clínico en infecciones del tracto respiratorio superior ocurrió en los años 50 (1).

Además de los efectos antimicrobianos de los macrólidos al inhibir la síntesis de proteínas por la unión a la subunidad 50s del ribosoma bacteriano, se ha documentado que tienen propiedades anti-inflamatorias e inmunomoduladoras. Esto ocurre por medio de la alteración en la producción de moco, la interferencia en la función de neutrófilos y macrófagos del tracto respiratorio, la alteración en los procesos de quimiotaxis, migración y activación celular, y en la disminución de la producción de citoquinas proinflamatorias $(1,2)$.

Dentro de las indicaciones aprobadas para el uso de los macrólidos se encuentra el manejo de la neumonía adquirida en la comunidad (NAC) donde se ha descrito tienen efectos inmunomoduladores independientes de la actividad antibacteriana (6). En estudios retrospectivos se observó que el tratamiento con macrólidos en adición a betalactámicos versus monoterapia con betalactámicos aumentó la sobrevida en estos pacientes (7). Adicional a esto, se observó que la estancia hospitalaria se redujo con el tratamiento con este grupo de antibióticos (8).

En el presente estudio describimos nuestra experiencia con el uso de claritromicina en pacientes críticamente enfermos para el tratamiento principalmente de NAC. Comparado con publicaciones previas que analizaron el riesgo de eventos cardiovasculares relacionados con el uso de claritomicina en este tipo de pacientes, el promedio de edad y la distribución por género es similar. En nuestro estudio la presencia de enfermedad cardiaca previa y enfermedad renal previa fue mayor a lo reportado en estudios previos (9, 10). Adicionalmente el puntaje de APACHE II, el puntaje de SOFA y los requerimientos de soporte ventilatorio y vasopresor en la UCI fueron altos, lo cual puede sugerir que nuestra población tiene una alta carga de comorbilidades y una mayor severidad de la enfermedad.

En los últimos 15 años se han reportado eventos cardiovasculares relacionados con su uso de los macrólidos. Entre estos está la prolongación del intervalo QT, el aumento de arritmias como torsades de pointes y taquicardia ventricular, y el mayor riesgo de muerte súbita de origen cardiaco (3-5). Estos efectos han sido más frecuentemente reportados con eritromicina, claritromicina y azitromicina $(3,11-13)$. Los factores relacionados con el potencial efecto arritmogénico 
de los macrólidos son secundarios a la repolarización ventricular retardada que es un proceso mediado por el flujo de salida intracelular de potasio $(14,15)$.

Se ha descrito que es más frecuente la presencia de los eventos adversos cardiovasculares asociados al uso de macrólidos al administrarlos simultáneamente con otros medicamentos que prolonguen el intervalo QT, además de la presencia de factores relacionados con la prolongación de intervalo QT como son el sexo femenino, edad avanzada, alteraciones electrolíticas como hipokalemia e hipomagnesemia, antecedente de insuficiencia cardiaca y bradicardia, entre otras $(15,16)$. Al realizarse un análisis ajustado por comorbilidades y características de los pacientes, se documenta que disminuye de 24 a $68 \%$ el riesgo de eventos cardiovasculares relacionados con el uso de claritromicina (17).

En nuestro estudio se reportó la presencia de hipokalemia en $13.89 \%$ de los pacientes y de hipomagnesemia en $11.11 \%$ de los pacientes. El medicamento usado de manera más frecuente concomitante a la claritromicina fue quetiapina con $28.95 \%$. Estos dos medicamentos se han asociado con la prolongación del intervalo QTc la cual puede ser mayor a 60 milisegundos en pacientes críticamente enfermos y al asociarse facilitan la presencia de arritmias tipo torsades de pointes $(18,19)$.

Por último, la frecuencia de arritmias tipo fibrilación o flutter auricular en nuestro estudio fue de $7.89 \%$ y de taquicardia ventricular $2.63 \%$. Sólo el $12.5 \%$ de los pacientes que fallecieron presentaron arritmia ventricular como causa de muerte. En estudios previos la frecuencia de arritmias fue de $6.5 \%$ en pacientes tratados con claritomicina lo cual es menor a lo encontrado en nuestros pacientes, y en un metaanálisis esto no se asoció a un mayor riesgo de mortalidad cardiovascular $(9,13)$.

\section{Conclusiones}

Los macrólidos son antibióticos que además de su acción antibacteriana pueden tener otros efectos benéficos para el tratamiento de enfermedades infecciosas. En nuestra experiencia, el uso de macrólidos se relacionó con una mayor frecuencia de arritmias con respecto a publicaciones previas, pero las complicaciones cardiovasculares no fueron la principal causa de muerte.

La presencia de múltiples comorbilidades, la severidad en las patologías de base y las combinaciones farmacológicas que estaban recibiendo los pacientes de nuestro estudio podrían explicar el aumento en la frecuencia de arritmias.

\section{Referencias}

1. Kwiatkowska B, Maślińska M. Macrolide Therapy in Chronic Inflammatory Diseases. Mediators Inflamm Mediators Inflamm. 2012; 636157.

2. Sevilla-Sánchez D, Soy-Muner D, Soler-Porcar N. Utilidad de los macrólidos como antiinflamatorios en las enfermedades respiratorias. Arch Bronconeumol. 2010; 46(5): 244-54.

3. Ray WA, Murray KT, Meredith S, Narasimhulu SS, Hall K, Stein CM. Oral erythromycin and the risk of sudden death from cardiac causes. $N$ Engl J Med. 2004; 351(11): 1089-96.

4. Abo-Salem E, Fowler JC, Attari M, Cox CD, Perez-Verdia A, Panikkath R, et al. Antibiotic-induced cardiac arrhythmias. Cardiovasc Ther. 2014; 32(1): 19-25.

5. Cheng Y-J, Nie X-Y, Chen X-M, Lin X-X, Tang K, Zeng W-T, et al. The Role of Macrolide Antibiotics in Increasing Cardiovascular Risk. J Am Coll Cardiol. 2015; 66(20): 2173-84.

6. Kovaleva A, Remmelts HHF, Rijkers GT, Hoepelman AIM, Biesma DH, Oosterheert JJ. Immunomodulatory effects of macrolides during communityacquired pneumonia: a literature review. J Antimicrob Chemother. 2012; 67(3): 530-40.

7. Martínez JA, Horcajada JP, Almela M, Marco F, Soriano A, García E, et al. Addition of a macrolide to a beta-lactam-based empirical antibiotic regimen is associated with lower in-hospital mortality for patients with bacteremic pneumococcal pneumonia. Clin Infect Dis Off Publ Infect Dis Soc Am. 2003; 36(4): 389-95.

8. Stahl JE, Barza M, DesJardin J, Martin R, Eckman MH. Effect of macrolides as part of initial empiric therapy on length of stay in patients hospitalized with community-acquired pneumonia. Arch Intern Med. 1999; 159(21): 2576-80.

9. Schembri S, Williamson PA, Short PM, Singanayagam A, Akram A, Taylor J, et al. Cardiovascular events after clarithromycin use in lower respiratory tract infections: analysis of two prospective cohort studies. BMJ. 2013; 346: f1235.

10. Chalmers JD, Singanayagam A, Murray MP, Hill AT. Prior statin use is associated with improved outcomes in community-acquired pneumonia. Am J Med. 2008; 121(11): 1002-1007.e1.

11. Ray WA, Murray KT, Hall K, Arbogast PG, Stein CM. Azithromycin and the risk of cardiovascular death. $N$ Engl J Med. 2012; 366(20): 1881-90.

12. Albert RK, Schuller JL. COPD Clinical Research Network. Macrolide antibiotics and the risk of cardiac arrhythmias. Am J Respir Crit Care Med. 2014; 189(10): 1173-80.

13. Gorelik E, Masarwa R, Perlman A, Rotshild V, Muszkat M, Matok I. Systematic Review, Meta-analysis, and Network Meta-analysis of the Cardiovascular Safety of Macrolides. Antimicrob Agents Chemother. 2018; 62(6): e00438-18.

14. Karmakar S, Padman A, Swamy Mane N, Sen T. Hypokalemia: a potent risk for QTc prolongation in clarithromycin treated rats. Eur J Pharmacol. 2013; 709(1-3): $80-4$.

15. Liu BA, Juurlink DN. Drugs and the QT interval - caveat doctor. N Engl J Med 2004; 351(11): 1053-6.

16. Shaffer D, Singer S, Korvick J, Honig P. Concomitant risk factors in reports of torsades de pointes associated with macrolide use: review of the United States Food and Drug Administration Adverse Event Reporting System. Clin Infect Dis Off Publ Infect Dis Soc Am. 2002; 35(2): 197-200.

17. Polgreen LA, Riedle BN, Cavanaugh JE, Girotra S, London B, Schroeder MC, et al. Estimated Cardiac Risk Associated With Macrolides and Fluoroquinolones Decreases Substantially When Adjusting for Patient Characteristics and Comorbidities. J Am Heart Assoc. 2018; 7(9).

18. Vieweg WVR, Hancox JC, Hasnain M, Koneru JN, Gysel M, Baranchuk A. Clarithromycin, QTc interval prolongation and torsades de pointes: the need to study case reports. Ther Adv Infect Dis. 2013; 1(4): 121-38.

19. Dube KM, DeGrado J, Hohlfelder B, Szumita PM. Evaluation of the Effects of Quetiapine on QTc Prolongation in Critically Ill Patients. J Pharm Pract. 2018; 31(3): 292-7. 